\title{
Tzanakis Scoring System in Acute Appendicitis Concern to Indian Population
}

\author{
Yeshwant Lamture' ${ }^{1}$, Rahul Y. Netragaonkar ${ }^{2}$, Varsha Gajbhiye ${ }^{3}$, Ranjeet Ambad ${ }^{4}$ \\ ${ }^{1}$ Professor, Dept. of General Surgery, J.N.M.C. Sawangi (Meghe) Wardha, ${ }^{2}$ Associate Professor, Dept. of \\ Community Medicine, DVVPF 'S Medical College, Ahmednagar, ${ }^{3}$ Asso. Professor in Pharmacology, ${ }^{4}$ Associate \\ Professor in Biochemistry, Datta Meghe Medical College. Nagpur
}

\begin{abstract}
Background: Acute appendicitis is amongst the most encountered emergencies seen in casualties requiring intervention. The incidence of negative laparotomy is $15 \%$ to $25 \%$ and is associated with notable morbidity. Scoring systems are useful and logical for distinguishing acute appendicitis from non-specific abdominal pain. Presently many scoring systems exist that aids in the diagnosis of acute appendicitis but still fail to decrease the rates of wrong diagnosis and the negative appendicectomy rate. This study has been undertaken to evaluate the efficacy of the Tzanakis scoring system for the Indian population in diagnosing acute appendicitis.
\end{abstract}

Method and Material: A prospective study to evaluate Tzanakis scoring system on 50 patients. The decision of an appendicectomy was taken by the consultant surgeon. The outcomes of the Tzanakis scoring system in terms of sensitivity, specificity, positive predictive value, negative predictive value, and diagnostic accuracy were calculated.

Results: The Tzanakis scoring system had sensitivity, specificity, PPV, NPV, and DA of $71.42 \%, 66.67 \%$, $83.33 \%, 50 \%$, and $70 \%$ respectively.

Conclusion: Tzanakis scoring system in diagnosing acute appendicitis has a lower value in Indian populations.

Keywords: Inflammation of the appendix, gangrene of appendix,perforation peritonitis.

\section{Introduction}

Acute appendicitis is amongst the many encountered emergencies seen in casualties requiring intervention. There is a $6 \%$ chance of appendicitis in the general population ${ }^{[1]}$. Though the mortality has declined from $26 \%$ to $1 \%$ with the dawn of broad-spectrum antibiotics and timely surgery, it remains 5 to $15 \%$ in the elderly ${ }^{[2]}$.

\section{Corresponding Author:}

\section{Dr. Rahul Y. Netragaonkar}

Associate Professor, Dept. of Community Medicine, DVVPF'S Medical College, Ahmednagar e-mail: dryrlamture@yahoo.co.in
The incidence of negative laparotomy is $15 \%$ to $25 \%$ and is associated with notable morbidity, the frequency being more in women of reproductive age group (till $45 \%$ ) due to the commonness of inflammatorytuboovarian diseases, ectopic pregnancy, and other gynecological pathology ${ }^{[3]}$. The delay in diagnosis may lead to rupture of the appendix in $17 \%$ to $40 \%$ of cases mostly in extremes of age. In the adolescents and elderly, it is linked with dramatic complications like an intraabdominal abscess, wound infection, and ultimately death ${ }^{[4]}$. Thus, confirmation of acute appendicitis is very crucial to achieve lower morbidity and mortality rates.

Ultrasonography (USG) with graded compressions has immense capacity in diagnosing appendicitis in its acute stage ${ }^{[5]}$. Various studies have been conducted on 
the utilization of these imaging systems. These studies reported a sensitivity of $75 \%$ to $94 \%$, a specificity of $86 \%$ to $100 \%$, and gross precision up to $96 \%{ }^{[6]}$. Its superiority over computed tomography is the absence of exposure to ionizing radiation thus helpful in pregnant and pediatric patients. The major disadvantage is that it is operator dependent leading to inter-observer bias and failure to see the appendix during the scan does not rule out the possibility of having an inflamed appendix ${ }^{[5]}$.

Despite the use of all these imaging techniques, the incidence of negative appendicectomy is not decreasing. These unmerited operations have a complication rate of approximately $13 \%$, which is nearby to that of an inflamed appendix. Removal of a healthy appendix has a mortality of $0.65 \%$. Protracted clinical observations aiming to reduce undesired operations may mean a delay in operations in $28 \%$ of cases and considerable danger of perforation ${ }^{[7] .}$

Scoring systems are useful and logical for distinguishing pain of acutely inflamed appendix from vague abdominal pain. Presently numerous scoring systems exist that aid in diagnosing acute appendicitis. However, these systems do not replace clinical acumen thereby just helping in the determination of acute appendicitis and aids in approaching a conclusion, whether a particular case should be operated or not, thus lowering the negative appendicectomy rate (NAR).

The original study done by Tzanakis et $\mathrm{al}^{[8]}$ in Greece reported sensitivity and specificity of $95.4 \%$ and $97.4 \%$ respectively. The diagnostic effectiveness of the score was $96.5 \%$ in his study. But in India, this scoring system was found inferior. For example, a comparative study between Tzanakis score and Alvarado score done by Shashikala $\mathrm{V}^{[9]}$ in India revealed that Tzanakis scoring system had a sensitivity of $79.62 \%$, a specificity of $83.3 \%$, PPV of $97.72 \%$, and NPV of $31.25 \%$ which was found better than Alvarado scoring system but still posing difficulties in diagnosis with a NAR of $12 \%$. Another study was done by Iqbal MM et $\mathrm{al}^{[10]}$ in Postgraduate medical center, Karachi, Pakistan revealed that the Tzanakis score had a sensitivity of $99 \%$, the specificity of $91 \%$, PPV of $99 \%$, and NPV of $91 \%$ with a diagnostic accuracy of $95 \%$. Similarly,A study done in Kathmandu Model Hospital, Nepal did by Sigdel GS et al ${ }^{111]}$ reveals that Tzanakis has a sensitivity of $91.48 \%$, a specificity of $66.66 \%$, and diagnostic accuracy of $90 \%$.
Despite various scoring systems and developments in the diagnostic and imaging modalities, the diagnosis of appendicitis is in dilemma which fails to decrease the rates of wrong diagnosis and the negative appendicectomy rate. This sequentially increases the cost for diagnosis by the use of expensive radiological modalities like Computed Tomography and MRI thus causing a delay in the treatment leading to an increase in morbidity and mortality of the patients.

This non-randomized prospective study has been undertaken to evaluate the effectiveness of the Tzanakis score in diagnosing acute appendicitis.

\section{Method}

The present study was undertaken in the department of surgery, Jawaharlal Nehru Medical College, Wardha in collaboration with Datta Meghe Medical College Hingana, Nagpur, Datta Meghe Institute of medical science (DMIMS), Sawangi, Meghe, Wardha, Maharashtra India.

Study Design: Prospective non-randomized study.

Study Population: All patients of acute appendicitis with age $>15$ years and $<60$ years.

Study Duration: July 2019-July 2020.

Sample Size: 50 patients

Inclusion Criteria: All patients with right lower quadrant pain and clinically diagnosed as acute appendicitis of age $>15$ years and $<60$ years.

\section{Exclusion Criteria:}

5. Patients with appendicular mass.

6. Patients of appendicitis with a known case of connective tissue disorder.

7. Patients with a past history of renal or ureteric stones and pelvic inflammatory disease.

\section{Pregnant women.}

Ethical clearance was obtained from the Ethics committee of DattaMeghe Institute of Medical Sciences (Deemed to be University).This prospective non-randomized study was conducted in Acharya VinobaBhave Rural Hospital, Sawangi. All the patients who fulfilled the eligibility criteria were subjected to routine hematological investigations, USG, and were scored based on Tzanakis scoring system. 
Tzanakis scoring system combines 4 variables as follows:

- Presence of right lower abdominal tenderness $=4$ points

- $\quad$ Rebound tenderness (Bloomberg sign $)=3$ points

- Laboratory findings: the presence of white blood cells greater than 12,000 in the blood $=2$ points

- Ultrasound findings: the presence of positive ultrasound scan findings $=6$ points

A score of 8 or more is suggestive of acute appendicitis requiring surgery ${ }^{[8]}$.
The decision to operate was taken by a consultant surgeon by clinical diagnosis with help of other appropriate investigations in special circumstances. All patients underwent appendicectomy with prior consent and the specimen was subjected to histopathological examination for confirmation of diagnosis. The minimum criteria for acute appendicitis were the appearance of neutrophils in mucosa, submucosa, and lamina propria ${ }^{[1]}$. The result of the Tzanakis score was calculated. The result was correlated with the findings obtained on histopathological examination and the data was analyzed using the necessary statistical calculations using SPSS 24.0 version, the results were then presented.

\section{Observations and Results:}

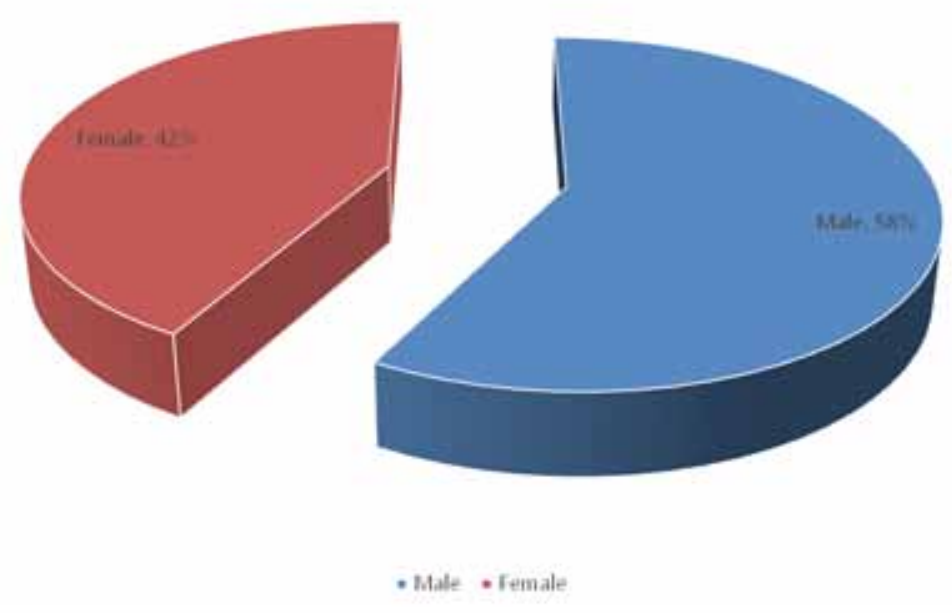

Graph No. 01: Gender

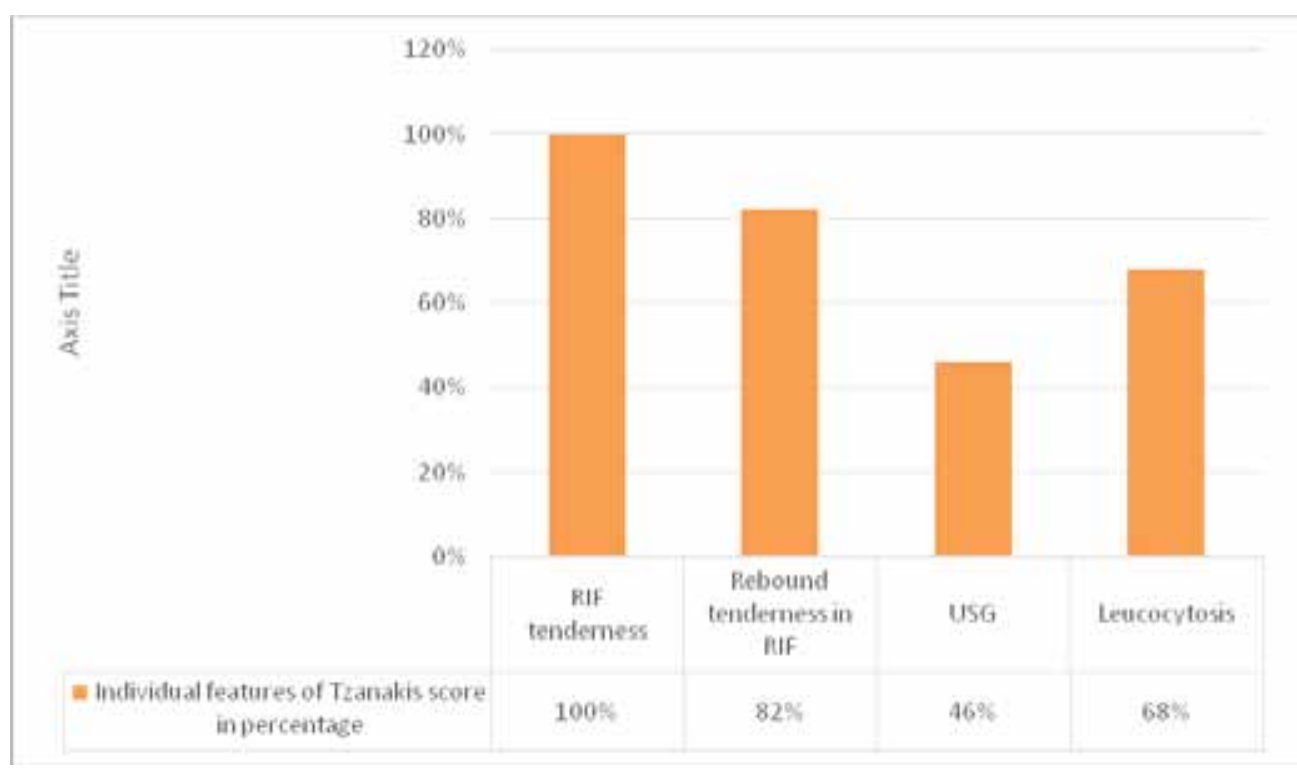

Graph 02: Individual characteristics of Tzanakis score : 


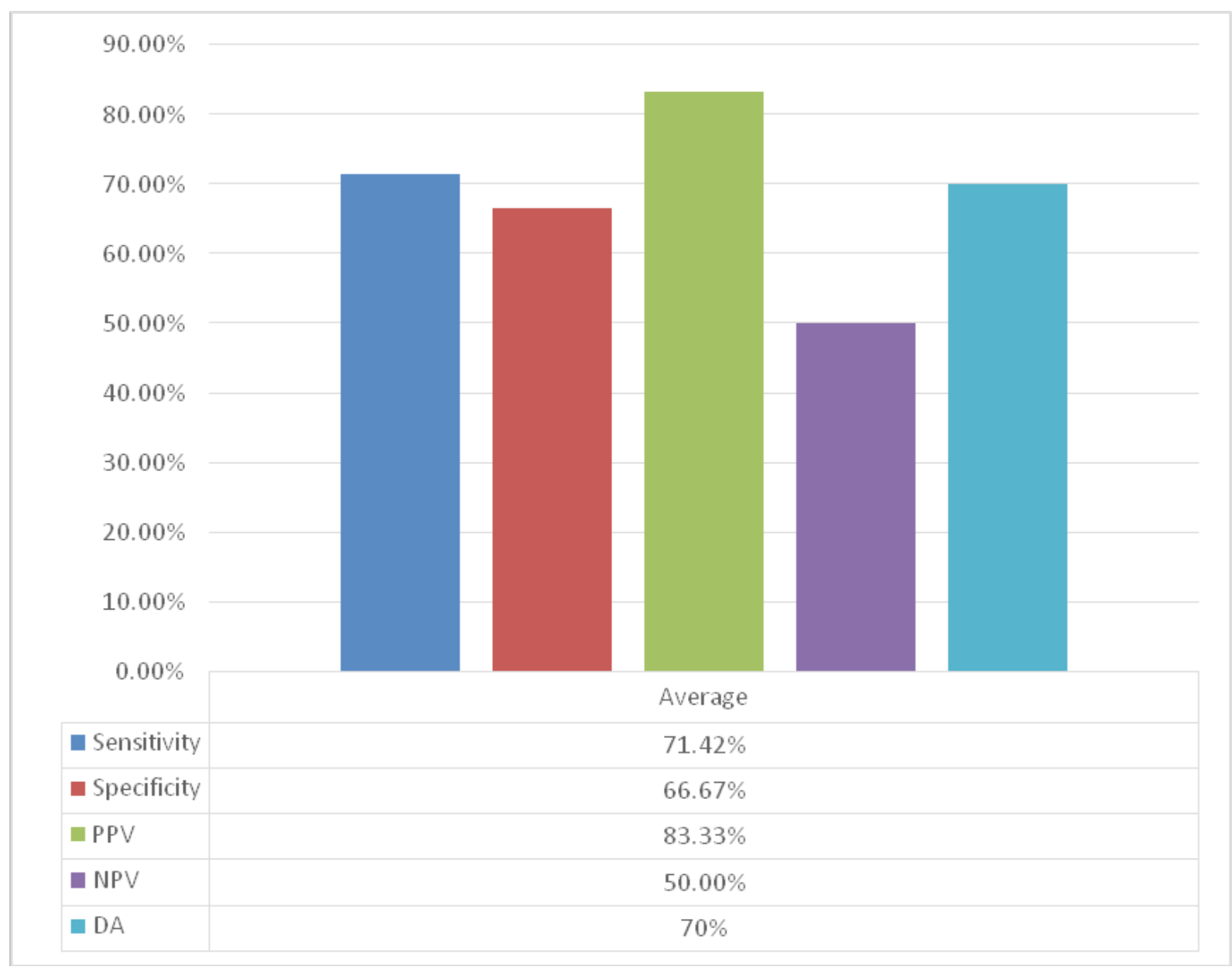

Graph 03: outcome of Tzanakis Scoring system

\section{Negative appendicectomy rate}

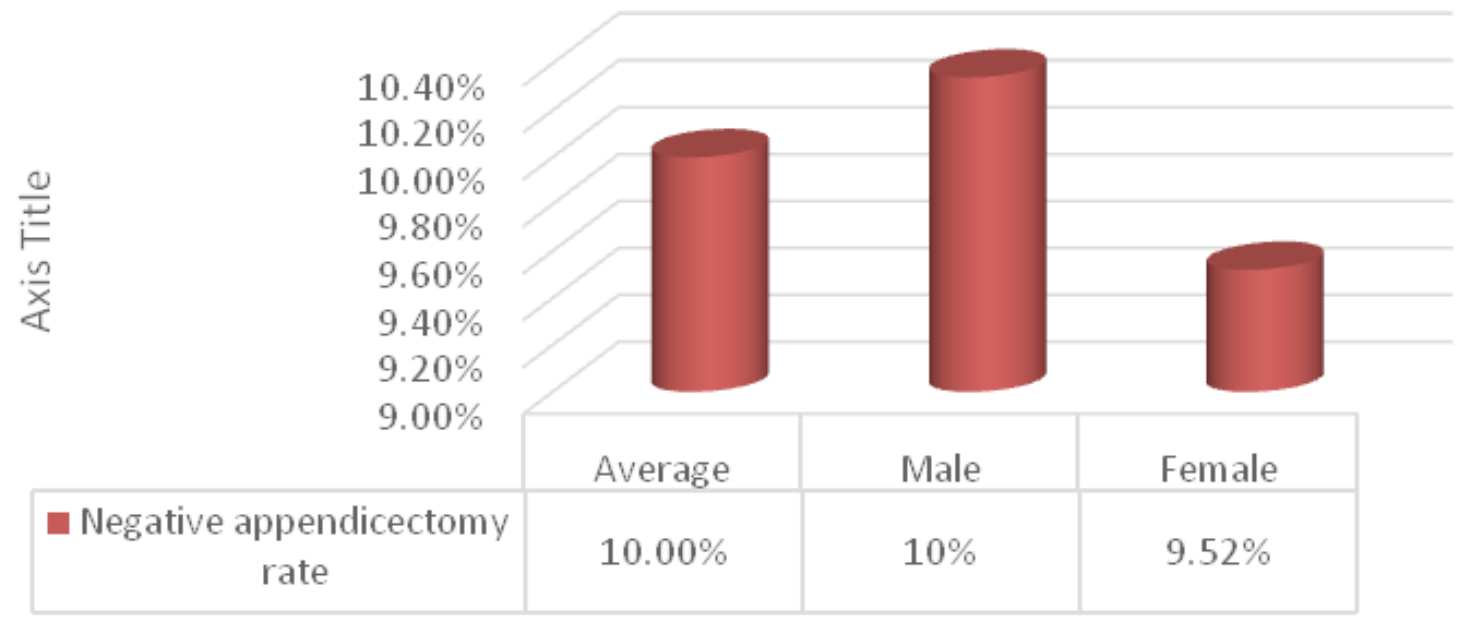

Graph 04: Negative appendicectomy rate of Tzanakis scoring system: 


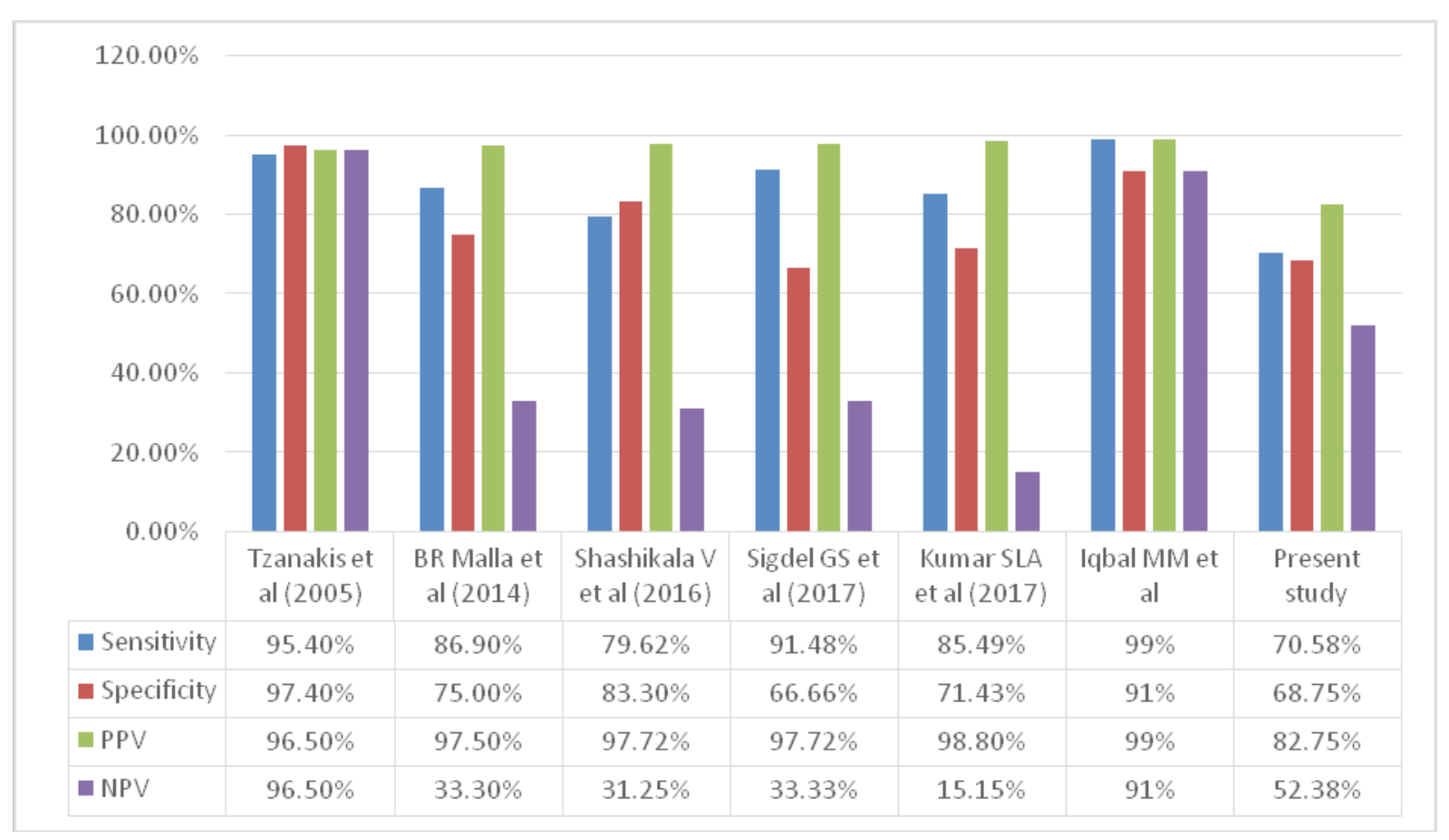

Graph 5: Comparison of final results of Tzanakis scoring system of various studies with our present study:

\section{Discussion}

Acute appendicitis continues to exist as the most widespread emergency in the world. A delay in making a diagnosis is associated with various complications which increases the morbidity and mortality in patients therefore a prompt and reliable diagnosis of acute appendicitis is mandatory. Furthermore, negative appendicectomy also accounts for the loss of financial resources and is associated with morbidity in 10 to $15 \%$ of cases.

Despite big breakthroughs in the imaging field, there remains uncertainty in diagnosing acute appendicitis due to atypical presentations of the disease. It has been repeatedly shown that investigations like USG lack specificity due to its operator dependency whereas investigations like CT scan and MRI are highly-priced demand more advanced equipment and competency. This makes a detailed clinical examination with primary investigations such as leucocyte count as the backbone in diagnosing acute appendicitis. This has compelled many surgeons to use diverse scoring systems for diagnosing acute appendicitis. The clinical evaluation is reliable in $50 \%$ to $80 \%$ of cases. The evaluation is more complex in the extremes of age and women of reproductive age group due to atypical presentations ${ }^{[12,13]}$.
The current study will deal with the evaluation of the Tzanakis scoring system to diagnose acute appendicitis in a simple, reliable, and cost-effective way thereby reducing the negative appendectomy rate and thus morbidity associated with it.

In this study, the sample population consisted of 50 patients out of which $58 \%$ were males and $42 \%$ were females (see graph 01). The male to female ratio in our study was found to be 1.4:1 which is comparable to the study done by Kumar SLA et al ${ }^{[14]}$ in which the male to female ratio was 1.6:1.

All the patients who accomplished the eligibility criteria were subjected to detailed clinical examination, routine hematological investigations, USG, and were scored based onthe Tzanakis scoring system. The decision to operate the patient including patients with a score less than the cut-off value was based on the clinical assessment and judgment taken by the consultant surgeon. All the patients underwent appendectomy with prior consent and the specimen was subjected to histopathological examination to confirm the diagnosis.

In our present study, 35 out of 50 patients $(70 \%)$ had inflamed appendix intra-operatively. Out of the remaining 15 patients, five patients had enterocolitis, 
three patients had Meckel's diverticulitis, three patients had the pelvic inflammatory disease (salpingitis), two patients had a ruptured ovarian cyst and two patients had inflamed mesenteric lymph node (with or without pus) as the intra-operative findings. Shashikala $\mathrm{V}$ et al ${ }^{[9]}$ in her study had five out of 50 patients with an alternative diagnoses; out of which one patient had enterocolitis and four patients had a pelvic inflammatory disease. Another study was done by Kumar SLA et al ${ }^{[14]}$ his study also reported six patients with alternative diagnoses in which three patients had salpingitis, two patients had an ovarian cyst and one patient had Meckel's diverticulitis.

Tzanakis et $\mathrm{al}^{[8]}$, in 2005 revealed a more comprehensible system to help in making the diagnosis of appendicitis. It includes four parameters comprising of specific signs, laboratory and radiological investigations such as right iliac fossa tenderness, rebound tenderness over the right iliac fossa, leucocytosis (WBC count $>12,000 / \mathrm{mm}^{3}$ ), and USG. A score of 8 or more is considered as acute appendicitis.

In our study, out of the four parameters present in the scoring system, tenderness in the right iliac fossa was the commonest sign seen in $100 \%$ of patients whereas rebound tenderness and leucocytosis was found in $82 \%$ and $68 \%$ of the patients respectively. USG showed features of appendicitis in only $46 \%$ of cases (see graph 02).

In the present study, 30 patients had a score of eight or more, and 20 patients who had a score of less than eightaccording to the Tzanakis score.

The 30 patients who scored eight or more had 25 patients with features of appendicitis on histopathology whereas 5 patients had a histologically normal appendix with no features of inflammation.

Similarly, 20 patients scored less than eight out of which there were 10 patients with a histologically normal appendix with no features of inflammation and 10 patients with features of appendicitis on histopathology.

The sensitivity, specificity, PPV, NPV, and diagnostic accuracy of the Tzanakis scoring system in our study was found to be $71.42 \%, 66.67 \%, 83.33 \%$, $50.00 \%$, and $70 \%$ respectively (see graph 03 ). The low sensitivity and specificity rate was due to the presence of high false-negative and false-positive results.
The study done by Tzanakis et al ${ }^{[8]}$ in 2005 had a sensitivity of $95.4 \%$, the specificity of $97.4 \%$, PPV of $96.5 \%$, NPV of $96.5 \%$, and diagnostic accuracy of $96.5 \%$ which is much higher as compared to our present study. This difference may occur due to low sample size, ethnic variation, and other demographic factors such as diet (see graph 5).

The study done by Shashikala V et al ${ }^{[9]}$ in 2016 reported a sensitivity of $79.62 \%$, a specificity of $83.3 \%$, PPV of $97.72 \%$, and NPV of $31.25 \%$ which were comparable to our present study. The study done by Iqbal MM et al ${ }^{[10]}$ in 2018 had results similar to that of Tzanakis et al ${ }^{[8]}$ with a sensitivity of $99 \%$, a specificity of $91 \%$, PPV of $99 \%$, NPV of $91 \%$, and diagnostic accuracy of $95 \%$ (see graph 5).

The study was done by Sigdel GS et al ${ }^{[11]}$ and Kumar SLA et al ${ }^{[14]}$ in 2017 had a specificity of $66.66 \%$ and $71.43 \%$ respectively which was comparable to our study whereas the NPV of both the studies was lower than our present study. Both studies had a sensitivity and PPV higher than our present study but the difference is not significant (see graph 5).

Similarly, the study done by BR Malla et al ${ }^{[15]}$ in 2014 had sensitivity, specificity, PPV, and NPV of $86.9 \%, 75 \%, 97.5 \%$, and $33.3 \%$ respectively. The outcome of the Tzanakis scoring system in the study conducted by Malla BR et al was comparable to our present study (see graph 5).

The negative appendicectomy rate (NAR) of the Tzanakis scoring system in the present study was found to be $10.34 \%$ percent in the males and $9.52 \%$ in females. The overall NAR observed for the Tzanakis scoring system was $10 \%$ (see graph no 04 ).

The negative appendicectomy rate in our present study for the Tzanakis Scoring system was comparable to NAR obtained in the studies done by Shashikala V et $\mathrm{al}^{[9]}(12 \%)$ and Iqbal MM et al ${ }^{[10]}(10.30 \%)$.

The disadvantage of the Tzanakis scoring system is that it does not include more clinical symptoms such as fever, nausea and vomiting, anorexia, migratory right iliac fossa pain, and supportive investigations such as CRP which augments the accuracy of the scoring system. Another factor for the low specificity of the Tzanakis score is that it gives the highest weightage to ultrasonography in its scoring system which has variable sensitivity and specificity due to inter-observer bias. 


\section{Conclusion}

- Similarly, the Tzanakis scoring system had sensitivity, specificity, PPV, NPV, and DA $71.42 \%$, $66.67 \%, 83.33 \%, 50.00 \%$, and $70 \%$ respectively.

- The overall negative appendicectomy rate of the Tzanakis scoring system was $10 \%$.

Ethical Clearance: Taken from institutional ethics committee.

Source of Funding: Self.

Conflict of Interest: Nil.

\section{References}

1. Lamture et al. anatomical variations related to position of appendix.journal of evolution of medical and dental sciences-JEMDS (2018)7 (46), 5030-5033.

2. Vaidya $\mathrm{V}$ et al. Reliability of leukocytosis in diagnosing acute appendicitis. J. Evolution Med. Dent. Sci. 2020;9(32):2274-2278,

3. Vaidya $\mathrm{V}$ et al. Migration of abdominal pain - an effective tool to identify appendicitis and the only parameter to screen. J Evolution Med Dent Sci 2020;9(33):2329-2333,

4. Mundada A et al. Anorexia in acute appendicitis: A non-specific factor with significant accuracy in diagnosis. Medical Science, 2020, 24(105), 28122816

5. Parks N, Schroeppel T. Update on imaging for acute appendicitis. Surg Clin North Am. 2011; 91(1):141-154.

6. Birnbaum B, Wilson S. Appendicitis at the millennium. Radiology. 2000; 215(2):337-348.

7. Khanal B, Ansari M, Pradhan S. Accuracy of ultrasonography in the diagnosis of acute appendicitis. Kathmandu Univ Med J (KUMJ). 2008;6(1):70-74.

8. Tzanakis Net al. A new approach to accurate diagnosis of acute appendicitis. World J Surg. 2005;29(9):1151-56.

9. Shashikala V. Comparative study of Tzanakis score vs Alvarado score in the effective diagnosis of acute appendicitis. Int J Biomed Adv Res. 2016;7(9):41820.

10. Iqbal $\mathrm{M}$ et al. Experience of Tzanakis Scoring System for Accurate Diagnosis of Acute Appendicitis in Jinnah Postgraduate Medical Centre, Karachi. Isra Med J. 2018; 10(1): 40-43.

11. Sigdel Get al. Tzanakis score vs Alvarado score in acute appendicitis. Department of Surgery, Kathmandu Model Hospital, Nepal, Department of surgery, Tribhuvan University Teaching Hospital, Kathmandu, Nepal. J Nepal Med Assoc. 2010;49(178):96-9.

12. Lamture Yet al. Clinicosonological and laboratory co-relation with histopathology of acute appendicitis to develop new diagnostic scoring system (Yash scoring system). IntSurg J. 2017;4:2556-64.

13. Chaudhari $Y$ et al. Prevalence of appendicitis at surgery inpatient department of a tertiary care hospital: A descriptive study. MedPulse International Medical Journal November. 2015; 2(11):768-770.

14. Kumar Set al. Evaluation of Tzanakis scoring system in acute appendicitis: a prospective study. IntSurg J. 2017;4:3338-43.

15. Malla B, Batajoo H. Comparison of Tzanakis Score vs Alvarado Score in the Effective diagnosis of acute Appendicitis. Kathmandu Univ Med J. 2014;45(1):48- 50. 